\title{
Phase behaviour of an ionic microemulsion system as a function of the cosurfactant chain length
}

\author{
W.K. Kegel and H.N.W. Lekkerkerker \\ Van't Hoff Laboratory, University of Utrecht, Padualaan 8, $3584 \mathrm{CH}$ Utrecht, The Netherlands
}

(Received 29 September 1992; accepted 17 February 1993)

\begin{abstract}
The phase behaviour of a microemulsion system consisting of equal volumes of brine and oil, sodium dodecyl sulphate (SDS) as surfactant and alcohols of different chain lengths (pentanol, hexanol and heptanol) as cosurfactant was studied. In the case of pentanol, at low surfactant concentrations and increasing alcohol concentration, the wcll-known progression of phase equilibria, Winsor I-Winsor III-Winsor II, was observed. The use of hexanol or heptanol as cosurfactant leads to rather different phase behaviour. At low surfactant concentrations and increasing alcohol concentration the following sequence of phase equilibria is observed: Winsor I-Winsor III-four-phase equilibrium (water-lamellar phase-isotropic microemulsion phase-oil)-Winsor III-Winsor II. Increasing the surfactant concentration starting from the four-phase equilibrium first leads to a three-phase equilibrium (lamellar phase-isotropic microemulsion-oil), then a two-phase equilibrium (lamellar-bicontinuous microemulsion) followed by a monophasic lamellar system. The observed change in phase behaviour as a function of the cosurfactant chain length is discussed in terms of phenomenological interfacial models of microemulsions.
\end{abstract}

Keywords: Cosurfactant chain length; ionic microemulsion; phase behaviour.

\section{Introduction}

Systems consisting of oil, water (without or with salt) and one or more surfactants exhibit a remarkably rich phase behaviour as a function of temperature, pressure and the concentrations of its components $[1,2]$. Under appropriate conditions, various microemulsion phases can form, ranging from random isotropic phases to more ordered ones.

Microemulsion systems can be composed of ionic or non-ionic surfactants. For systematic phase behaviour studies, simple three-component water-oil-non-ionic surfactant microemulsions are

Correspondence to: H.N.W. Lekkerkerker, Van't Hoff Laboratory, University of Utrecht, Padualaan 8, $3584 \mathrm{CH}$ Utrecht, The Netherlands. more convenient than their complicated ionic counterparts which typically consist of five components: water, oil, surfactant, cosurfactant and salt. There seems to be a notion that all the characteristic aspects of microemulsion phase behaviour are already present in the non-ionic microemulsion systems, and thus from a fundamental point of view there appears to be little incentive to study the complex ionic microemulsion systems, which require a much larger experimental effort to control the relevant parameters. Nevertheless, as will become clear from the work presented in this paper, there are cases where the latter systems apparently allow a wider range of relevant parameters to be probed, leading to interesting phase behaviour which (so far) has not been observed in non-ionic microemulsion systems.

We first briefly recapitulate some aspects of the 
phase behaviour of non-ionic systems and their theoretical interpretation, which are relevant for the work on ionic microemulsions reported here. The phase behaviour of non-ionic surfactant systems has been studied extensively by Kahlweit and co-workers $[1,3]$. A cut through the phase diagram in the temperature-surfactant concentration plane at an oil-to-water ratio of about unity displays a characteristic "fish-like" shape schematically illustrated in Fig. 1. The body of the fish present at relatively low surfactant concentrations corresponds to the stability region of the Winsor III equilibrium, i.e. a random (possibly bicontinuous) microemulsion phase in equilibrium with excess water and oil. Variation of the temperature gives rise to Winsor I (oil droplets in water in equilibrium with excess oil) and Winsor II (water droplets in oil in equilibrium with excess water) equilibria. At increasing surfactant concentrations, the volume of the middle phase of the Winsor III equilibrium swells at the expense of the water and oil phase. This eventually gives rise to a monophasic microemulsion system which, upon further increase of the surfactant concentration, may undergo a firstorder transition to a lamellar phase.

Systematic variation of the surfactant chain length has revealed [3] that with increasing chain length the body of the fish shrinks, i.e. the Winsor III stability region becomes smaller with respect to both the temperature range and the surfactant concentration range. Also, the first-order microemulsion-lamellar phase transition occurs at a lower surfactant concentration. As the latter decrease proceeds faster than the shrinking of the body of the fish, the range of surfactant concentrations for which the system is a monophasic microemulsion becomes smaller.

The above features of the phase behaviour have been successfully explained by a simple phenomenological interfacial model $[4,5]$. In this model the microemulsion is treated as a collection of surfactant monolayers separating water and oil domains. The properties of these monolayers are controlled by the curvature energy [6]

$$
F_{\mathrm{c}}=\int_{A}\left[(K / 2)\left(c_{1}+c_{2}-2 c_{0}\right)^{2}+\bar{K} c_{1} c_{2}\right] \mathrm{d} A
$$

where $K$ and $\bar{K}$ are the elastic moduli associated with the mean and gaussian curvature, $A$ denotes the area, $c_{0}$ is the spontaneous curvature, and $c_{1}$ and $c_{2}$ are the principal curvatures of the monolayer. It is assumed that the temperature controls $c_{0}$ and that $K$ is increased by increasing the

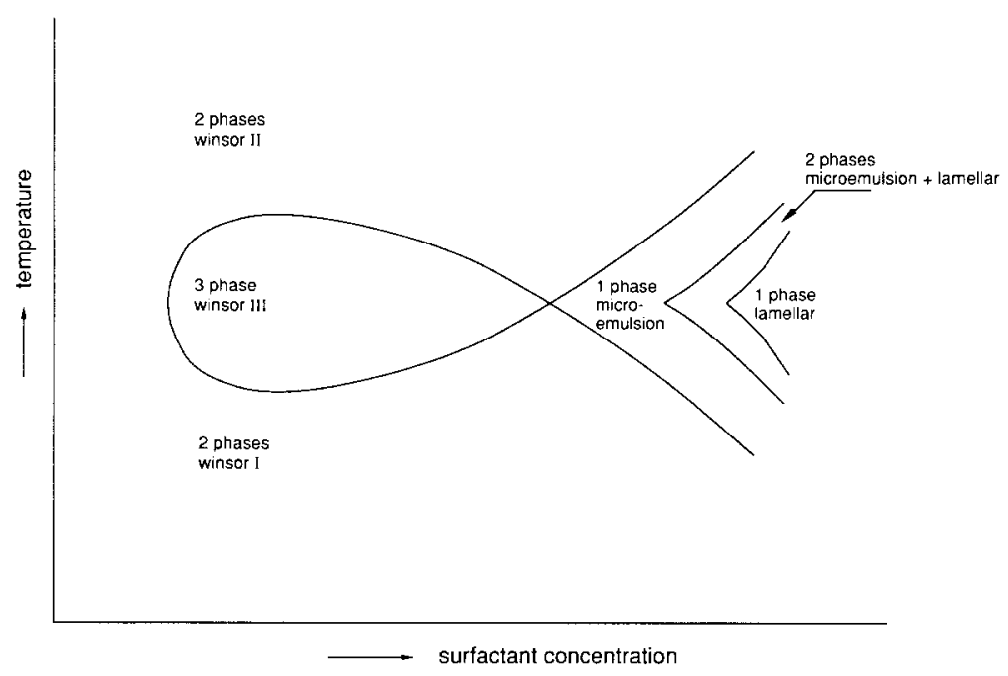

Fig. 1. Schematic representation of a typical phase diagram of a non-ionic system in the temperature-surfactant concentration plane for an oil:water ratio of 1 . 
surfactant chain length; $\bar{K}$ remains zero in this model. Theories relating $K$ and $\bar{K}$ to monolayer properties are referred to in the discussion section. The interfacial model produces a three-phase coexistence between water-rich and oil-rich phases and a disordered microemulsion phase. Lamellar phases are also described by this model. With increasing $K$, the lamellar phase becomes more stable and the first-order microemulsion-lamellar phase transition occurs at smaller surfactant concentrations. Eventually the range of surfactant concentrations for which the system is a monophasic microemulsion vanishes at a lamellar-microemulsion-water-oil multiple point. A refined version of the interfacial model [7] (but again keeping $\bar{K}=0$ ) predicts that for still higher $K$ values there is now a three-phase coexistence between the water, oil and the lamellar phase, i.e. the lamellar phase replaces the random microemulsion phase in the body of the fish. Exactly the same phase behaviour scenario is predicted by a microscopic lattice theory of microemulsions [8].

In the above treatment, the middle-phase microemulsion is effectively considered as a molten lamellar phase. However, there is experimental evidence [9] that the microemulsion phase can be thought of as a disordered analogue of a bicontinuous cubic phase [10]. If the middle-phase microemulsion indeed resembles a molten cubic phase rather than a molten lamellar phase it will contain a large density of "handles" or passages. The elastic modulus $\bar{K}$ will then play an important role, since according to the Gauss-Bonnet theorem

$\int c_{1} c_{2} \mathrm{~d} A=4 \pi\left(N_{\mathrm{c}}-N_{\mathrm{h}}\right)$

where $N_{\mathrm{c}}$ denotes the number of (unconnected) components and $N_{\mathrm{h}}$ the number of "handles" or passages. This implies, together with Eqn (1), that a negative $\bar{K}$ will have a destabilizing effect on the middle-phase microemulsion; thus the loss of stability of the microemulsion phase relative to the lamellar phase with increasing cosurfactant chain length could also be triggered by a decreasing $\bar{K}$.

As far as we are aware the replacement of the microemulsion phase by the lamellar phase in the body of the fish has not been reported for threecomponent water-oil-non-ionic surfactant systems, but it has been observed in four-component ionic microemulsion systems [11]. Even more interestingly, Hackett and Miller [12] observed a four-phase water-oil-microemulsion-liquid crystal (possibly lamellar) equilibrium in the five-component system brine, sodium dodecyl sulphate (SDS), hexanol and decane. However, in a system containing pentanol and cyclohexane instead of hexanol and decane, only the "classical" Winsor I, III and II equilibria were observed at low surfactant concentrations [13].

In order to investigate the appearance of this four-phase equilibrium more systematically, we decided to study the phase behaviour of an ionic microemulsion system with cosurfactants of different chain length. We selected a system consisting of equal volumes of brine (water plus $\mathrm{NaCl}$ ) and oil (cyclohexane plus alcohol) with SDS as surfactant and alcohols of different chain lengths (pentanol, hexanol, heptanol) as cosurfactant. In a recent study [14] it was shown that the composition of the mixed surfactant-cosurfactant monolayer between brine and cyclohexanc did not change when pentanol was replaced by hexanol. The phase behaviour of the SDS plus pentanol system, which has been investigated extensively in our laboratory [13], shows a typical fish shape with the role of the temperature in non-ionic microemulsion systems replaced by the concentration of cosurfactant or salt. By increasing the alcohol chain length we find the same trend as in systems containing non-ionic surfactants with increasing surfactant chain length but now the lamellar phase appears to penetrate into the body of the fish, giving rise to a four-phase equilibrium consisting of an isotropic microemulsion phase, a lamellar phase and excess brine and oil phases. Increasing the surfactant concentration starting from the four-phase equilibrium first leads to a three-phase equilibrium (lamellar-isotropic microemulsion-oil), then a two-phase equilibrium (lamellar-isotropic microemulsion) followed by a monophasic lamellar system. This change in phase 
behaviour as a function of the cosurfactant chain length is accompanied by an increase in the dispersion size $\xi$ in the microemulsion middle phase indicative of an increase in $K$ [15].

After describing the experimental procedures and results in the next two sections, the observed phase behaviour is discussed in terms of the bending elastic parameters of the mixed surfactantcosurfactant monolayer at the oil/water interface in the final section.

\section{Experimental}

\section{Materials}

Sodium dodecyl sulphate was "specially pure" grade purchased from BDH. Cyclohexane, $n$-pentanol, $n$-hexanol, $\mathrm{NaCl}$ (Baker) and $n$-heptanol (Fluka) were "analysed" grade. All chemicals were used without further purification. Deionized water was doubly distilled before use.

\section{Preparation of the samples}

Portions $(8.00 \mathrm{~g}$, and in a number of cases $4.00 \mathrm{~g})$ of the initial oil phase were carefully poured onto $10.00 \mathrm{~g}$ (or $5.00 \mathrm{~g}$ ) of the initial water phase in glass tubes with Teflon-sealed screw caps. The initial oil phase was a mixture of different weight fractions of $n$-pentanol, $n$-hexanol or $n$-heptanol and cyclohexane. The water phase was composed of $0.20 \mathrm{M}$ $\mathrm{NaCl}$ and various amounts of SDS. Equilibrium was attained by gently rolling the samples on a roller bench (about $1 \mathrm{rev}^{-1} \mathrm{~min}^{-1}$ ) for several days. Subsequently the samples were permitted to equilibrate at constant temperature $\left(25 \pm 0.1^{\circ} \mathrm{C}\right)$ until no change in phase volumes was observed.

Depending on the alcohol used, the time necessary to obtain complete phase separation varied from less than a week (pentanol) to more than a year (heptanol).

\section{Characterization of birefringent phases}

Birefringent phases were characterized using polarization microscopy. The observations were made with a Leitz Metallux 3 optical microscope equipped with a Linkam THMS 600 hot-stage element. The qualification "lamellar phase" is reserved for birefringent phases showing a focal conics texture [16].

\section{Determination of the dispersion size in microemulsion middle phases}

In the pentanol system the dispersion size $\xi$ in the microemulsion middle phase was obtained from the middle-phase volume $V_{\mathrm{m}}$ and the total area $A$ in the system $A=N_{\mathrm{s}} \sigma_{\mathrm{s}}$ where $N_{\mathrm{s}}$ and $\sigma_{\mathrm{s}}$ are the number of SDS molecules and the area per molecule respectively, using

$\xi=6 \phi_{\mathrm{o}} \phi_{\mathrm{w}} \frac{V_{\mathrm{m}}}{A}$

where $\phi_{\mathrm{o}}$ and $\phi_{\mathrm{w}}$ are the volume fractions of oil and water respectively. In the hexanol and heptanol systems, $\xi$ was determined from the mass fractions of SDS, $x_{\mathrm{s}}$ in the microemulsion middle phase (in equilibrium with a lamellar phase) using

$\xi=\frac{6 \phi_{\mathrm{o}} \phi_{\mathrm{w}} M_{\mathrm{w}}}{N_{\mathrm{av}} x_{\mathrm{s}} \rho_{\mathrm{m}} \sigma_{\mathrm{s}}}$

where $M_{\mathrm{w}}, N_{\mathrm{av}}$ and $\rho_{\mathrm{m}}$ are the molar weight of SDS, Avogadro's number and the density of the microemulsion middle phase, respectively. The mass fraction $x_{\mathrm{s}}$ was determincd by evaporating the liquid components from a (weighed) sample. Correction for the amount of $\mathrm{NaCl}$ was carried out. The volume fraction $\phi_{0}$ was measured with a Packard Model 433 gas chromatograph equipped with a flame ionization detector and a glass column packed with cross-linked polystyrene resin. From $\phi_{0}, x_{\mathrm{s}}$ and the density of SDS (i.e. $1.45 \mathrm{~g} \mathrm{~cm}^{-3}$ [13]) $\phi_{\mathrm{w}}$ was calculated. In all the systems, we found $\phi_{0} \approx \phi_{\mathrm{w}} \approx 0.5$. Further, we used $\rho_{\mathrm{m}}=$ $0.90 \mathrm{~g} \mathrm{~cm}^{-3}$ and $\sigma_{\mathrm{s}}=0.93 \mathrm{~nm}^{2}$ as obtained for the pentanol and the hexanol systems [14]. 


\section{Results}

Figure 2 shows the phase diagrams in the surfactant concentration-cosurfactant concentration plane of the microemulsion systems with equal volumes of the water phase $(0.2 \mathrm{M} \mathrm{NaCl}$ and SDS $)$ and oil phase (cyclohexane and alcohol) for different alcohols. SDS concentrations and alcohol concentrations are given as weight fractions in the initial water and oil phase.

In the case of pentanol (Fig. 2(a)) at low SDS concentrations (less than $0.042 \%(\mathrm{w} / \mathrm{w})$ in the initial water phase) and increasing cosurfactant concentration, the well-known progression of phase equilibria Winsor I-Winsor III-Winsor II is observed. Increasing the SDS concentration starting from the Winsor III region along the path A-B indicated in Fig. 2(a) (assumed to correspond to a "balanced microemulsion", i.e. $c_{0}=0$ ) leads to a single-phase microemulsion system between 0.042 and $0.078 \%$ $(w / w)$. At still higher surfactant concentrations $(0.078-0.085 \%(\mathrm{w} / \mathrm{w}))$ a first-order phase transition to a lamcllar phase takes place. Finally above $0.085 \%(\mathrm{w} / \mathrm{w})$ the system is monophasic lamellar. On decreasing or increasing the cosurfactant concentration in the lamellar system, first-order transitions to the $\mathrm{L}_{1}$ phase (oil droplets in water) and the $L_{2}$ phase (water droplets in oil) respectively are observed.

The use of hexanol (Fig. 2(b)) instead of pentanol leads to rather different phase behaviour. The amount of cosurfactant in the system necessary to reach the middle-phase region (i.e. the region inbetween Winsor I and Winsor II phase equilibria) is reduced and the concentration range over which it is stable becomes significantly smaller. At low SDS concentrations (less than $0.02 \%(\mathrm{w} / \mathrm{w})$ ) and increasing hexanol concentration the following sequence of phase equilibria is observed: Winsor IWinsor III-four-phase equilibrium (waterlamellar phase-microemulsion phase-oil)-Winsor III-Winsor II. In the four-phase equilibrium region, the volume of the lamellar phase first increases, reaches a maximum and then decreases upon increasing the cosurfactant concentration while the total volume of the surfactant-rich phases remains approximately constant. Increasing the SDS concentration starting from the middle of the middle-phase region along the path $\mathrm{A}-\mathrm{B}$ as indicated in Fig. 2(b), the volumes of the surfactantrich phases increases at the expense of the excess phases. The lamellar phase primarily takes up the water phase forming a threc-phase equilibrium (lamellar-microemulsion-excess oil) somewhat above $0.02 \%(\mathrm{w} / \mathrm{w})$ SDS, a two-phase equilibrium (lamellar-microemulsion) at about $0.035 \%(\mathrm{w} / \mathrm{w})$ SDS and a single lamellar phase above $0.048 \%$ (w/w) SDS.

The heptanol systems (Fig. 2(c)) appeared to be extremely viscous, which may be the reason for the long time necessary for the lamellar phase to separate. Because of these huge equilibration times, we limited ourselves to low surfactant concentrations. Here again, as in the case of hexanol, four-phase equilibria (brine-lamellar phase-microemulsion phase-oil) were observed in the middle of the middle-phase region.

In Table 1 the dispersion size $\xi$ determined from Eqns (3) and (4) for the different cosurfactants in the microemulsion middle phases is presented. For the calculation of $\xi$ in the heptanol system, the molecular area $\sigma_{\mathrm{s}}$ as obtained for the pentanol and the hexanol systems was used. There is a steep increase on changing from pentanol to hexanol and a less prominent difference between heptanol and hexanol.

\section{Discussion}

We note that increasing the alcohol chain length in the brine-cyclohexane-SDS-cosurfactant system has a dramatic influence on the phase behaviour. We now discuss our results in terms of phenomenological interfacial models of microemulsion phase behaviour $[4,5,7,10]$, limiting ourselves to balanced systems, i.e. systems with $c_{0} \approx 0$. As mentioned in the Introduction, the common feature of the interfacial models is that they relate microemulsion phase behaviour to the bending elastic moduli $K$ and $\bar{K}$ of the extended surfactant 

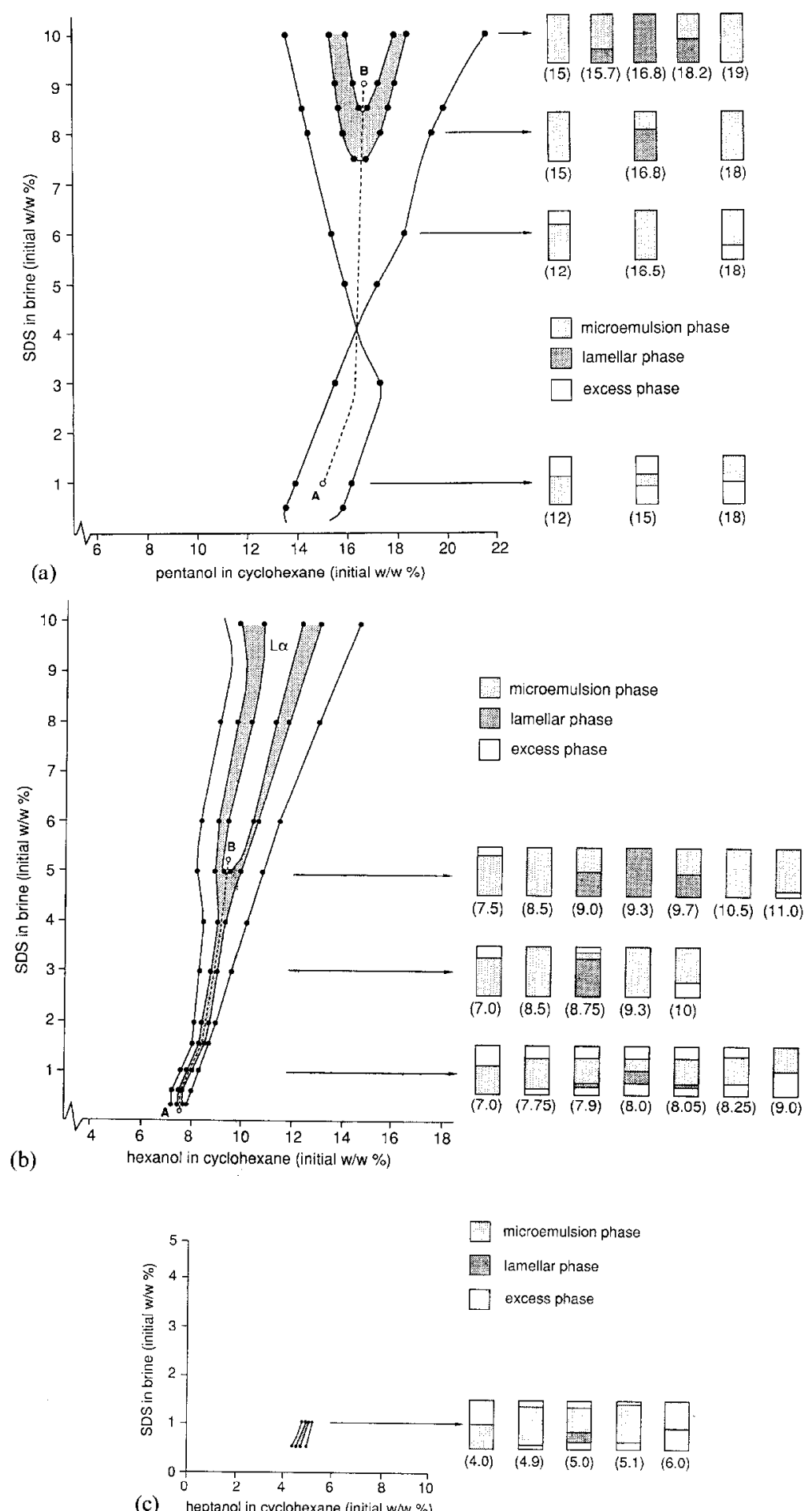

(c) heptanol in cyclohexane (initial w/w\%)

Fig. 2. Phase behaviour of brine $(0.2 \mathrm{M} \mathrm{NaCl})$, cyclohexane, SDS and cosurfactant in the SDS-cosurfactant concentration plane with equal volumes of the brine and oil phases as a function of cosurfactant chain length: (a) pentanol; (b) hexanol; (c) heptanol. The shaded regions in the phase diagrams correspond to a lamellar phase in equilibrium with one or more isotropic phases. The pictograms are on scale representations of the relative volumes of the coexisting phases. The cosurfactant concentrations are indicated between parentheses. 
TABLE I

Dispersion size $\xi$ in the middle-phase microemulsions as a function of the cosurfactant chain length ${ }^{\mathrm{a}}$

\begin{tabular}{ll}
\hline Cosurfactant & $\xi(\mathrm{nm})$ \\
\hline Pentanol & 35.8 \\
Hexanol & 71.5 \\
Heptanol & 92.1 \\
\hline
\end{tabular}

${ }^{a}$ All sizes were measured in the microemulsion middle phases at an initial SDS concentration of $0.01 \%(w / w)$ in the water phase.

monolayer separating the water and oil domains. Therefore we first consider the expected changes in the bending elastic moduli upon increasing the chain length of the cosurfactant.

Safinya et al. [17] presented experimental evidence (obtained using high resolution $\mathrm{X}$-ray scattering) that $K$ increases with cosurfactant chain length in an SDS-cosurfactant bilayer system in water. We assume that the same trend holds in a monolayer system as a function of the cosurfactant chain length. This assumption is further corroborated by the work of Binks et al. [15] who presented experimental evidence that the microemulsion dispersion size increases with $K$ in a wide variety of surfactant systems. The data in Table 1 show that in the systems studied here, $\xi$ increases when changing from pentanol to hexanol to heptanol, from which we conclude that $K$ increases in this order. In contrast to the situation for $K$ there are no experimental values for $\bar{K}$ with increasing cosurfactant chain length. However, the behaviour of $\bar{K}$ can be inferred from the lateral pressure profile. As shown by Helfrich [18], $\bar{K}$ is the negative second moment of the lateral profile $\Pi(z)$ :

$\bar{K}=-\int_{-\infty}^{+\infty} z^{2} \Pi(z) \mathrm{d} z$

Since both the electric double layer and the surfactant plus cosurfactant chains make a positive contribution to $\Pi(z)[19-21]$ we deduce that $\bar{K}<0$ for a surfactant monolayer at the oil/water interface. Statistical mechanical calculations [19] show that the lateral pressure at the chain side increases with cosurfactant chain length. Therefore we expect that $\bar{K}$ becomes more negative when changing from pentanol to hexanol. This argument is valid only if changes in the cosurfactant chain length do not modify the film composition. While in general this will be the case [22], we have recently shown [14] that the SDS : cosurfactant ratio at the cyclohexane/brine interface remains constant when pentanol is replaced by hexanol. To conclude, we think there is strong evidence that $K$ will increase upon increasing the cosurfactant chain length while $\bar{K}$ becomes more negative. With this in mind, we now discuss our results in terms of the interfacial models referred to above.

As mentioned in the Introduction, we can distinguish two points of view in the theoretical treatment of lamellar-microemulsion competition. Starting from the molten lamellar point of view for the microemulsion phase, the observed change in phase behaviour upon changing the cosurfactant from pentanol to hexanol can be explained by assuming that this leads to such an increase in $K$ that the so-called "polyphasic" region predicted by the simple version of the interfacial model [5] is entered. This region corresponds to "complex polyphasic" equilibria between water, oil, lamellar phase, and the microemulsion phases with a waterto-oil ratio different from unity [5]. Unfortunately, no further details about this region are given. The more refined model of Golubovic and Lubensky [7] predicts for a given temperature that the fourphase oil-water-microemulsion-lamellar equilibrium, which is stable for a single value of $K$, is followed by a three-phase oil-water-lamellar equilibrium for still higher values of $K$. We observe four-phase oil-water-microemulsion-lamellar equilibria both for hexanol and heptanol as a cosurfactant. Assuming that $K$ increases in changing from hexanol to heptanol, our observation implies that four-phase equilibria exist for a range of $K$ values above a certain minimum. However, the model of Golubovic and Lubensky is designed for a three-component system rather than the fivecomponent system we are dealing with here, which 
has obvious consequences for the dimension of the four-phase coexistence region.

In the second approach, where the microemulsion is considered as a molten cubic phase, the transition is triggered by a decreasing $\bar{K}$ rather than an increasing $K$. As argued above, $\bar{K}$ will become more negative with increasing cosurfactant chain length. Consequently the stability of the lamellar phase will increase relative to the microemulsion phase if the latter indeed contains a large number of "handles".

Finally, the interfacial models discussed here only include topological interactions between the surfactant monolayers but neglect the usual colloidal interactions [23] such as van der Waals and electrostatic interactions. These interactions may play an important role and at the present time it is not clear whether this neglect of the colloidal interactions is permitted. In this work, clear experimental indication of their role is found in the asymmetric swelling behaviour of the lamellar phase in the hexanol system.

To conclude, depending on the assumed nature of the microemulsion middle phase (i.e. molten lamellar or molten cubic) an increase in $K$ and a decrease in $\bar{K}$ with the cosurfactant chain length both qualitatively explain the loss of stability of the microemulsion phase (leading to four-phase equilibria). Although we found a clear indication that interactions other than topological may play a role in the system studied here, there is no need to invoke them to explain our observations at this (qualitative) stage. More experimental and theoretical work is required to decide whether the dominant effect leading to four-phase equilibria is an increase in $K$ or a decrease in $\bar{K}$ or whether it is a combination of both effects.

\section{Acknowledgements}

The authors are indebted to E.J. Vlietstra (Department of Physical Organic Chemistry) for his kind help with the polarization microscopy technique. The investigation was supported by the Netherlands Foundation of Chemical Research (SON) with financial aid from the Netherlands Organization for Scientific Research (NWO).

\section{References}

1 M. Kahlweit, R. Strey and G. Bussse, J. Phys. Chem., 94 (1990) 3881.

2 A.M. Bellocq, J. Biais, P. Bothorel, B. Clin, G. Fourche, P. Lalanne, B. Lemaire, B. Lemanceau and D. Roux, Adv. Colloid Interface Sci., 20 (1984) 167.

3 M. Kahlweit, R. Strey and P. Firman, J. Phys. Chem., 90 (1986) 671 .

4 D. Andelman, M.E. Cates, D. Roux and S.A. Safran, J. Chem. Phys., 87 (1987) 7229.

5 M.E. Cates, D. Andelman, S.A. Safran and D. Roux, Langmuir, 4 (1988) 802.

6 W. Helfrich, Z. Naturforsch., Teil C, 28 (1973) 693.

7 L. Golubovic and T.C. Lubensky, Phys. Rev. A., 41 (1990) 41 .

8 G. Gompper and M. Schick, Phys. Rev. B., 41 (1990) 9148.

9 R. Strey, J. Winkler and L. Magid, J. Phys. Chem., 95 (1991) 7502.

10 G. Porte, J. Appell, P. Bassereau and J. Marignan, J. Phys. (Paris), 50 (1989) 1335.

11 B.P. Binks, H. Kellay and J. Meunier, Europhys. Lett., 16 (1991) 53.

12 J.L. Hackett and C.A. Miller, SPE Res. Eng., 3 (1988) 791

13 P.L. De Bruyn, J.Th.G. Overbeek and G.J. Verhoeckx, J. Colloid Interface Sci., 127 (1989) 244.

14 W.K. Kegel, G.A. van Aken, M.N. Bouts, H.N.W. Lekkerkerker, J.Th.G. Overbeek and P.L. De Bruyn, Langmuir, 9 (1993) 252.

15 B.P. Binks, O. Abillon and D. Langevin, Langmuir, 5 (1989) 415.

16 N.H. Hartshorne and A. Stuart, Crystals and the Polarizing Microscope, Edward Arnold, London, 1970.

17 C.R. Safinya, E.B. Sirota, D. Roux and G.S. Smith, Phys. Rev. Lett., 62 (1989) 1134.

18 W. Helfrich, in R. Balian, M. Kléman and J.P. Poirier (Eds), Les Houches, Session XXXV, 1980, Physics of Defects, North Holland, Amsterdam, 1981, p. 715.

19 I. Szleifer, D. Kramer, A. Ben-Shaul, W.M. Gelbart and S.A. Safran, J. Chem. Phys., 92 (1990) 6800.

20 H.N.W. Lekkerkerker, Physica A, 167 (1990) 384.

21 M. Winterhalter and W. Helfrich, J. Phys. Chem., 92 (1988) 6865.

22 T. Ahsan, R. Aveyard and B.P. Binks, Colloids Surfaces, 52 (1991) 339 .

23 J.N. Israelachvili, Intermolecular and Surface Forces, 2nd edn, Academic Press, London, 1991. 